\title{
Fatores que influenciam na continuidade da colaboração entre equipes de projetos desenvolvidos em eventos de curta duração
}

\author{
Tiago H. S. Leite ${ }^{1}$, Fernando Figueira Filho ${ }^{1}$, Leandro de Almeida Melo ${ }^{1}$, \\ Cleidson R. B. de Souza ${ }^{2}$, Fábio Freire da Silva Junior ${ }^{1}$ \\ ${ }^{1}$ DIMAp - Departamento de Informática e Matemática Aplicada - \\ Universidade Federal do Rio Grande do Norte (UFRN) \\ Caixa Postal 1524 - 59078-970 - Natal - RN - Brasil \\ ${ }^{2}$ Instituto de Ciências Exatas e Naturais - Universidade Federal do Pará (UFPA) \\ 66055-090 - Belém - PA - Brasil \\ \{tiagoleite, leandroalmeida, fabiofreire\}@ppgsc.ufrn.br, \\ fernando@dimap.ufrn.br, cleidson.desouza@acm.org
}

\begin{abstract}
Time-bounded collaborative events such as hackathons and game jams involve project teams that collaborate for a short period of time. Previous research explored various aspects of these events, but the factors that contribute or hinder successful collaboration among project team members are still unkown. In this paper, we present preliminary findings from of an exploratory study with twenty participants of two hackathons and one game jam. These findings show positive and negative factors that contribute to the continuity of collaboration among event team members.
\end{abstract}

Resumo. Eventos de curta duração, tais como hackathons e game jams, envolvem equipes de projeto que colaboram por um curto período de tempo. As pesquisas anteriores exploraram vários aspectos desses eventos, mas os fatores que contribuem ou atrapalham uma colaboração bem-sucedida entre membros de equipes de projeto são ainda desconhecidos. Neste artigo, são apresentados os resultados preliminares de um estudo exploratório com vinte participantes de dois grandes hackathons e um game jam. Os resultados preliminares apontam fatores positivos e negativos que contribuem para continuidade da colaboração entre membros de equipes de projetos desenvolvidos durante eventos de curta duração.

\section{Introdução}

Eventos de curta duração têm chamado muita atenção da comunidade científica da área de CSCW [Filippova et al. 2017]. Dois tipos de eventos desta natureza são os hackathons e as game jams, que estão dentro de um grupo com diversos modelos distintos de organização de eventos de curta duração, i.e. data dives, codefests, hack-days, dentre outros.

Nesse contexto, as game jams estão se tornando bem populares [Kultima 2015]. Os participantes deste tipo de evento basicamente se organizam em equipes e trabalham longas horas em um problema ou tema específico para desenvolver protótipos de jogos. 
Já os hackathons são eventos de curta duração onde um grupo de pessoas se reúne para criar protótipos e/ou aplicativos funcionais [Warner and Guo 2017].

A literatura que aborda esta temática é recente e está aumentando em diversas áreas de estudo. Entretanto, ainda pouco se conhece sobre o que ocorre após esses eventos, em particular, os fatores que contribuem para continuação ou não de um projeto iniciado durante uma game jam ou hackathon. O entendimento do que influencia na continuidade da colaboração entre membros de equipe de um projeto é importante para compreender como projetar sistemas que ofereçam suporte para colaboração entre participantes de eventos de curta duração.

Neste artigo, são reportados os resultados de estudo preliminar baseado em entrevistas com vinte participantes de dois hackathons e uma game jam. Três fatores foram destacados como positivos para continuidade da colaboração após o término de um evento: (i) manter contato com os participantes;(ii) encontrar-se pessoalmente com os colegas de equipe; (iii) criar startups com novas iniciativas. Três fatores foram destacados como negativos: (i) falta de investimento para continuar com os projetos; (ii) abandono dos projetos por diversos motivos; (iii) necessidade de refazer todo o projeto. Além disso, fatores ambíguos também foram destacados, entretanto, serão elucidados em trabalhos futuros. O artigo introduz a literatura relacionada na Seção 2, a metodologia na Seção 3, os resultados na Seção 4, e as considerações finais na Seção 5.

\section{Eventos de curta duração}

Eventos de curta duração são organizados em vários formatos e propósitos, com adaptações em diversos domínios do conhecimento, que indicam que tais eventos são uma forma intensiva de trabalho colaborativo. Eles podem ser aplicados para a aprendizagem informal e colaborativa, criação de startups, protótipos inovadores para artes e cultura, inovação cívica aberta e o fortalecimento das interações em domínios científicos específicos, como a biologia computacional [Filippova et al. 2017].

Estes eventos também atraem e conectam pessoas com interesses em comum, que compartilham o mesmo local ou não, podendo ser à distância, que incentiva os participantes a debater conjuntamente sobre problemas de interesse compartilhado em um ambiente colaborativo [Filippova et al. 2017, Pe Than et al. 2018]. Além disso, eles permitem que as melhores ideias cheguem ao topo, incentivando o modelo rápido de protótipos por meio de um ambiente informal com menos expectativa de sucesso comercial imediato, que assume o risco de falhas, induzindo a criatividade através de restrições [Preston et al. 2012, Pe Than et al. 2018].

\section{Metodologia}

Este estudo tem natureza exploratória e descritiva, tendo como objetivo identificar o que acontece com os projetos desenvolvidos em eventos de curta duração e como as relações que foram construídas durante estes eventos se comportam.

O estudo exploratório foi desenvolvido em duas etapas, sendo na primeira a realização de um questionário com participantes de eventos de curta duração e a segunda com realização de entrevistas com participantes dos mesmos eventos. As perguntas que guiaram as entrevistas foram as seguintes:(i) $\mathrm{O}$ que aconteceu com as relações sociais 
após o término do evento? (ii)Há planos em continuar trabalhando com a mesma equipe após o fim do evento? (iii) Há continuidade do projeto desenvolvido após o fim do evento?

A primeira coleta de dados foi realizada em dois hackathons, organizados em duas grandes cidades do Brasil, com participação in loco apenas no primeiro. Ambos os eventos foram organizados por uma grande empresa, porém foram de natureza cívica. Um convite foi enviado por e-mail para sessenta contatos que foram fornecidas pelos organizadores dos eventos para fins de pesquisa. Cerca de 46 participantes responderam ao convite e, destes, treze se dispuseram para a realização de entrevistas.

Um segundo convite para entrevistas foi enviado para os participantes de uma grande game jam de escala global. A base de contatos dos participantes era disponibilizada no site da organizadora do evento e foi coletada de forma automática por meio de um script. Convites para entrevista foram enviados para cem pessoas selecionadas de forma aleatória a partir de uma amostra de cerca de mil contatos. Dessas cem pessoas, sete participaram das entrevistas.

Todo o contato realizado (online) com o entrevistado foi gravado e transcrito sob autorização do próprio, apenas para fins acadêmicos e, sendo assim, os dados serão apresentados de forma anônima. Foram realizadas entrevistas semi estruturadas com participantes de diversos estados do Brasil, sem interferência de terceiros, apenas com o participante da pesquisa e o entrevistador. O tempo de cada entrevista variou de forma razoável, com média de 34 minutos. Os participantes foram questionados sobre o que aconteceu com as relações construídas durante o evento, se elas se mantiveram, se os projetos foram continuados e quais as dificuldades encontradas nesse contexto.

A partir das entrevistas que foram realizadas, a análise dos dados se deu nos seguintes passos: (i) transcrição das entrevistas, (ii) codificação dos dados e (iii) escrita de memorandos. Todas as etapas, com exceção de algumas transcrições, foram executadas pelo autor do estudo e supervisionadas pelo orientador, com o objetivo de manter a confiabilidade do processo de análise.

Para a realização da análise e codificação dos dados foi utilizada uma metodologia baseada na Teoria Fundamentada nos Dados (ou Grounded Theory ou, abreviando, TFD).

\section{Resultados parciais}

Nesta seção serão apresentados os temas que surgiram da pesquisa exploratória e da análise das entrevistas realizadas em todos os eventos. A maioria desses temas que surgiram na pesquisa se tornaram mais claros nas entrevistas e, diante disso, muitas das afirmações serão trazidas em forma de sentenças. Cada trecho foi representado como [P\#], onde o número do participante é indicado, pois todos serão tratados de forma anônima. Além disso, os resultados serão apresentados como pontos positivos, pontos negativos e ambíguos.

\subsection{Pontos Positivos}

A maioria dos entrevistados afirmaram que continuam mantendo contato com participantes do evento após o seu fim, seja com toda a equipe ou com parte dela. Além disso, há relatos de que manter o contato com os participantes é algo recorrente, como é possível identificar na seguinte fala: "Dos cinco eventos que participei, em três eu man- 
tive o contato com os participantes da equipe. Neste último evento, mantivemos a mesma equipe" [P7].

Outro participante relata que a granularidade da equipe faz com que o evento seja o momento ideal para que todos possam se encontrar pessoalmente, já que cada um tem uma rotina diferente e isso impacta na dificuldade de haver encontros presenciais: "São pessoas que eu mantenho contato e a gente pode participar de outros eventos, (...), cada um tem suas atividades e as pessoas são bem atarefadas e é preciso um evento para juntar todo mundo" [P1].

Uma das informações que chamou atenção foi a equipe que se formou no evento e, após o fim do mesmo, criaram uma startup e iniciaram outros projetos nesta nova iniciativa: "Sim, ainda estamos com a startup até hoje e com as outras equipes ainda tenho contato também, já trabalhamos em outros projetos e temos contato pessoalmente com eles" [P7].

\subsection{Pontos Negativos}

Um dos pontos negativos encontrados é quando um participante afirma que não há planos em trabalhar com a mesma equipe, pois ele fala que se houvesse investimento seria uma realidade possível: "Eu gostaria de trabalhar com eles, eu achei uma equipe boa para trabalhar, mas a gente não tem investimento e por enquanto não daria" [P9].

As respostas obtidas não ficaram muito claras sobre quais são os objetivos dos participantes para com seus projetos, mas pelas citações há indícios de que alguns projetos estejam sendo abandonados ou descontinuados, por diferentes motivos e, muitas vezes, sem uma justificativa: "Não, sinceramente não apareceu nenhuma oportunidade pra gente continuar com o projeto" [P16].

Entretanto, nos poucos casos em que houveram respostas claras, o participante descreveu o planejamento dos próximos passos do projeto: "Sim, Resolvemos fazer uma nova versão dele. Estamos reimplementando tudo, toda a codificação. Estamos vendo a possibilidade de colocar numa loja, porém com ele todo refatorado. Estamos trabalhando, meio lento, mas estamos desenvolvendo sim" [P1]. Isso indica que houve a necessidade de refazer todo o projeto para que o ele fosse publicado.

\subsection{Ambíguos}

Nesta subseção, foram indicados os achados onde não se classificaram em pontos positivos nem em pontos negativos. Tais destaques serão validados em trabalhos futuros.

Um dos participantes afirma que boa parte das equipes são formadas por pessoas que já se conheciam antes do evento: "Um deles é meu amigo pessoal, amigo meu há anos. Eu mantenho contato com ele sim" [P9].

Mais da metade dos entrevistados indicam que pretendem trabalhar com a mesma equipe: "A gente sempre fica esperando abrir evento de hackathons para poder se encontrar de novo e trabalhar e desenvolver ideias novas que a a gente vai tendo ao decorrer do ano" [P10].

Alguns participantes relataram que pretendem dar continuidade ao projeto, porém aguardam feedback das empresas organizadoras sobre alguns pontos do projeto para 
dar sequência às atividades: "A gente tem o contato sim, mas na expectativa de ter esse retorno da empresa, para que esse trabalho do evento não morra" [P11].

\section{Discussão}

Em relação aos hackathons, estudos que indicam a intenção em abandonar os projetos desenvolvidos nestes eventos. Estes estudos mostram que "pelo fato do projeto não ter sido finalizado durante o evento, ele não foi continuado" [Gama 2017]. Nas entrevistas realizadas em nosso estudo, não houve perguntas direcionadas sobre a intenção de abandono do projeto. Mesmo assim, os resultados indicaram que há indícios de que estes projetos estão sendo abandonados, e que este é um ponto negativo a ser observado nesses tipos de eventos. Trainer (2016) também mostra a "relação entre o fato do projeto não ter sido concluído durante o evento e a sua descontinuidade". Mais uma vez, esses resultados encontrados ratificam parte dos nossos achados, onde os participantes, mesmo indicando que há interesse em continuar trabalhando com a mesma equipe, em muitos casos, não indicaram pretensão em continuar com o desenvolvimento dos projetos do evento.

[Trainer et al. 2016] indica, em seus estudos, que "muitos dos participantes gostariam de encontrar os colegas em eventos futuros". Ele também fala que eles "estreitaram os laços sociais, criando grupos em redes sociais e trocando informações que vão além do evento", o que corrobora com nossos achados, onde grande parte dos participantes afirmaram que mantém contato com outros companheiros que participaram do evento e indicaram o desejo de encontrá-los novamente, o que foi destacado como um ponto positivo.

Nolte (2018) relata, em seus achados, que muitos dos "participantes indicaram intenção em continuar o desenvolvimento dos projetos que trabalharam durante o hackathon, mas destaca que estes projetos, que foram continuados, realizaram um bom planejamento antes do evento". Em nossa análise, menos da metade dos participantes se mostraram dispostos em continuar com o desenvolvimento dos projetos, o que difere dos resultados de [Nolte et al. 2018] e abre caminho para aprofundar mais sobre as razões pelas quais estes participantes não pretendem continuar com os projetos.

As contribuições de [Reng et al. 2013] também indicam que muitos "participantes não demonstram interesse em dar continuidade aos jogos após o fim do evento, relacionando esta descontinuidade ao fato dos participantes não terem chegado a um nível satisfatório do jogo, demonstrando desinteresse com o próprio". Em nossos achados, mais da metade dos participantes entrevistados afirmaram que não tinham pretensões em continuar com o projeto desenvolvido ou não sabiam informar, o que foi considerado um ponto negativo.

Os próximos passos deste estudo propõe um entendimento mais amplo sobre o "pós-evento", mais precisamente sobre o que acontece com as relações sociais e os projetos desenvolvidos. Entretanto, ainda há necessidade de direcionamento por quais dimensões de análise devem ser abordadas, para que uma nova coleta de dados seja realizada de forma mais direcionada, possibilitando melhores resultados.

\section{Agradecimentos}

Os autores gostariam de agradecer ao $\mathrm{CNPq}$ pelo apoio financeiro através dos processos 420801/2016-2, 311256/2018-0, 1580018 e 1738626. 


\section{Referências}

Filippova, A., Chapman, B., Geiger, R. S., Herbsleb, J. D., Kalyanasundaram, A., Trainer, E., Moser, A., and Stoltzfus, A. (2017). Hacking and making at time-bounded events: Current trends and next steps in research and event design. In Companion of the 2017 ACM Conference on Computer Supported Cooperative Work and Social Computing, CSCW' 17 Companion, pages 363-370, New York, NY, USA. ACM.

Gama, K. (2017). Preliminary findings on software engineering practices in civic hackathons. In 2017 IEEE/ACM 4th International Workshop on CrowdSourcing in Software Engineering (CSI-SE), pages 14-20.

Kultima, A. (2015). Defining game jam. In FDG.

Nolte, A., Pe-Than, E. P. P., Filippova, A., Bird, C., Scallen, S., and Herbsleb, J. D. (2018). You hacked and now what?: - exploring outcomes of a corporate hackathon. Proc. ACM Hum.-Comput. Interact., 2(CSCW):129:1-129:23.

Pe Than, E. P. P., Herbsleb, J., Nolte, A., Gerber, E., Fiore-Gartland, B., Chapman, B., Moser, A., and Wilkins-Diehr, N. (2018). The 2nd workshop on hacking and making at time-bounded events: Current trends and next steps in research and event design. In Extended Abstracts of the 2018 CHI Conference on Human Factors in Computing Systems, CHI EA '18, pages W35:1-W35:8, New York, NY, USA. ACM.

Preston, J. A., Chastine, J., O'Donnell, C., Tseng, T., and MacIntyre, B. (2012). Game jams: Community, motivations, and learning among jammers. International Journal of Game-Based Learning (IJGBL), 2(3):51-70.

Reng, L., Schoenau-Fog, H., and Kofoed, L. B. (2013). The motivational power of game communities-engaged through game jamming. In Proceedings of the 8th International Conference on the Foundations of Digital Games, pages 14-17.

Trainer, E. H., Kalyanasundaram, A., Chaihirunkarn, C., and Herbsleb, J. D. (2016). How to hackathon: Socio-technical tradeoffs in brief, intensive collocation. In Proceedings of the 19th ACM Conference on Computer-Supported Cooperative Work \& Social Computing, CSCW'16, pages 1118-1130, New York, NY, USA. ACM.

Warner, J. and Guo, P. J. (2017). Hack.edu: Examining how college hackathons are perceived by student attendees and non-attendees. In Proceedings of the 2017 ACM Conference on International Computing Education Research, ICER '17, pages 254262, New York, NY, USA. ACM. 